\title{
Erratum to: Significance of IL-6 in the transition of hormone-resistant prostate cancer and the induction of myeloid-derived suppressor cells
}

\author{
Chun-Te Wu • Ching-Chuan Hsieh • Cheng-Chia Lin • \\ Wen-Cheng Chen • Ji-Hong Hong • Miao-Fen Chen
}

Published online: 24 April 2013

(C) Springer-Verlag Berlin Heidelberg 2013

Erratum to: J Mol Med (2012) 90:1343-1355

DOI 10.1007/s00109-012-0916-x

A mistake occurred in Figure 6a; the panel for TRAMP-HR was erroneously duplicated. Accordingly,
Figure 6a has been replaced with a corrected version of the figure as shown below. The authors claim that this correction does not influence the conclusion of the study and would like to apologize for this oversight.

The online version of the original article can be found at $\mathrm{http}$ //dx.doi.org/ 10.1007/s00109-012-0916-x.

\section{C.-T. Wu $\cdot$ C.-C. Lin}

Department of Urology, Chang Gung Memorial Hospital,

Keelung, Taiwan

C.-T. Wu • C.-C. Hsieh • W.-C. Chen · J.-H. Hong • M.-F. Chen

College of Medicine, Chang Gung University, Taoyuan, Taiwan

C.-C. Hsieh

Department of General Surgery, Chang Gung Memorial Hospital,

Chiayi, Taiwan

W.-C. Chen • M.-F. Chen $(\square)$

Department of Radiation Oncology, Chang Gung Memorial

Hospital, Chiayi, Taiwan

e-mail: miaofen@adm.cgmh.org.tw

J.-H. Hong

Department of Radiation Oncology, Chung Gung Memorial

Hospital, Linkou, Taiwan 
(A)

IL6 silencing vector

TRAMP-C1

TRAMP-HR

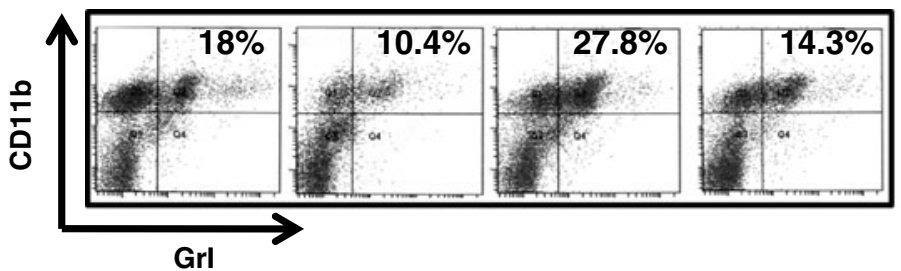

TRAMP-C1

TRAMP-HR

IL-6

DAPI
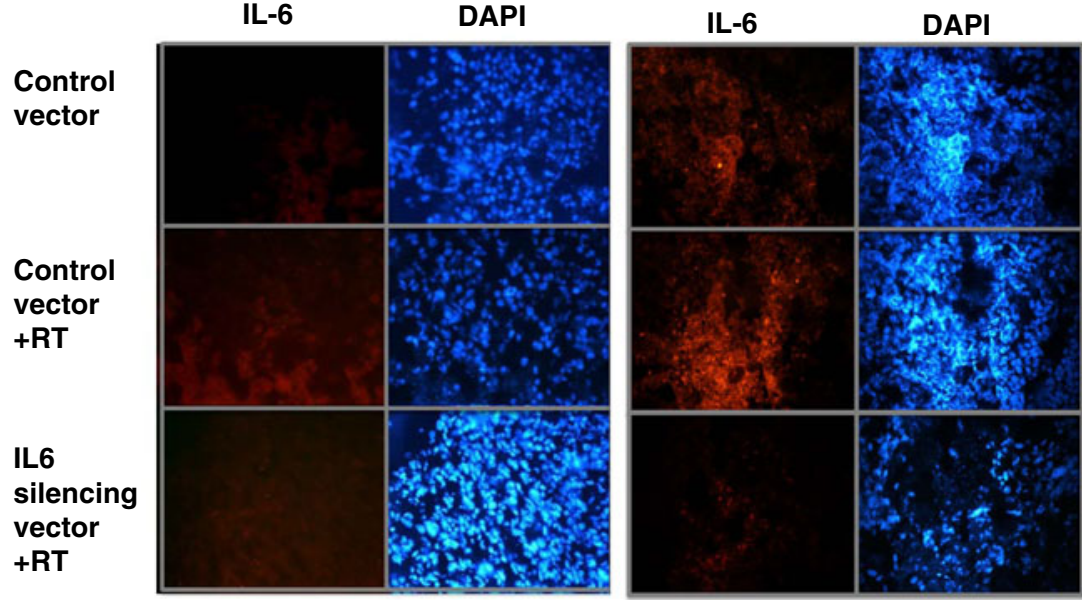